\title{
Frontières
}

\section{Rupture dans la ligne du temps : la souffrance chez les femmes âgées atteintes d'un cancer incurable et leur interprétation du temps}

\section{Valérie Bourgeois-Guérin}

Volume 25, numéro 1, automne 2012

Le vieillissement et sa diversité

URI : https://id.erudit.org/iderudit/1018234ar

DOI : https://doi.org/10.7202/1018234ar

Aller au sommaire du numéro

Éditeur(s)

Université du Québec à Montréal

ISSN

1916-0976 (numérique)

Découvrir la revue

Citer cet article

Bourgeois-Guérin, V. (2012). Rupture dans la ligne du temps : la souffrance chez les femmes âgées atteintes d'un cancer incurable et leur interprétation du temps. Frontières, 25(1), 127-151. https://doi.org/10.7202/1018234ar
Résumé de l'article

Des écrits montrent que des liens étroits unissent la souffrance et l'interprétation du temps chez les femmes âgées et les personnes atteintes de cancer. La définition de la souffrance de Ricoeur (1994) et les constats d'autres penseurs nous ont poussés à tenter de comprendre comment la souffrance des femmes âgées atteintes d'un cancer incurable se lie à leur interprétation du temps. Pour cela, nous avons fait une recherche qualitative, fondée sur les témoignages de femmes âgées atteintes d'un cancer incurable. Les résultats dévoilent que l'attente et l'annonce du cancer sont des événements du passé, souffrants à vivre. Plusieurs vivent leur présent au jour le jour, profitent du moment et de leurs capacités actuelles pour ne pas penser au futur, source de désespoir et/ou associé à la mort. Cette étude contribue à redéfinir, à mieux questionner et comprendre la souffrance des femmes âgées atteintes d'un cancer incurable. 


\section{RUPTURE DANS LA LIGNE DU TEMPS : LA SOUFFRANCE CHEZ LES FEMMES ÂGÉES ATTEINTES D'UN CANCER INCURABLE ET LEUR INTERPRÉTATION DU TEMPS}

Valérie Bourgeois-Guérin Psychologue, Programme des personnes âgées, Hôpital Pierre Janet,

RÉSUMÉ

Des écrits montrent que des liens étroits unissent la souffrance et l'interprétation du temps chez les femmes âgées et les personnes atteintes de cancer. La définition de la souffrance de Ricœur (1994) et les constats d'autres penseurs nous ont poussés à tenter de comprendre comment la souffrance des femmes âgées atteintes d'un cancer incurable se lie à leur interprétation du temps. Pour cela, nous avons fait une recherche qualitative, fondée sur les témoignages de femmes âgées atteintes d'un cancer incurable. Les résultats dévoilent que l'attente et l'annonce du cancer sont des événements du passé, souffrants à vivre. Plusieurs vivent leur présent au jour le jour, profitent du moment et de leurs capacités actuelles pour ne pas penser au futur, source de désespoir et/ou associé à la mort. Cette étude contribue à redéfinir, à mieux questionner et comprendre la souffrance des femmes âgées atteintes d'un cancer incurable. 
Some writings indicate that suffering and the interpretation of time of older women and people with cancer could be linked. The definition of suffering of Ricœur (1994) and the findings of other thinkers led us to try to understand how the suffering of older women with terminal cancer is linked to their interpretation of time. For this, we made a qualitative research based on interviews realized with older women with terminal cancer. The results reveal that the waiting and the announcement of cancer are events of the past linked to their suffering. Many of these women live their present day by day, enjoy the present moment and their current capabilities, not to think about the future, source of despair and / or associated with death. This study helps to redefine, question and understand the suffering of older women suffering from incurable cancer.

MOTS CLÉS: Temps - souffrance- cancer - vieillissement - femmes mort

KEYWORDS: Time- suffering- cancer- aging- women- death

\section{VIEILLIR AU FÉMININ \\ ET ÊTRE ATTEINTE \\ D'UN CANCER INCURABLE}

Le fait de vieillir s'inscrit dans le temps, dans la durée. Être âgée est une expression qui renvoie directement au temps qui s'est écoulé. Or, comme le soulève De Beauvoir (1970, p.301): «que le déroulement du temps universel ait abouti à une métamorphose personnelle, voilà ce qui nous déconcerte. » Comme le soulève Bersay (2005, p.41):

Nous sommes dans une situation contradictoire. D'un côté, nous souhaitons vivre longtemps, de l'autre nous ne voulons pas être vieux pour ne pas nous tenir à proximité de la mort. 
Cette contradiction face au vieillissement s'inscrit dans un rapport au temps tronqué où le désir de longévité est couplé au refus de vieillir.

Dans ce contexte social, le vieillissement est souvent mal perçu, mal accueilli. Cette réalité est encore plus évidente lorsque I'on se penche sur les conceptions sociales du vieillissement au féminin (Charpentier et al., 2010; De Beauvoir, 1970; Grenier et al., 2007). En effet, la vision des femmes âgées véhiculée dans la société nord-américaine actuelle est souvent très négative, péjorative (Charpentier et al., 2010 ; Grenier, 2002). Le vieillissement du corps des femmes, notamment, est dévalorisé et il n'est pas rare qu'il soit ouvertement combattu (Covan, 2005; Garner, 1999). Les risques d'infantilisation sont aussi accrus chez les femmes âgées dont le rôle est souvent dévalorisé (Nelson, 2002). Ces réalités peuvent évidemment venir engendrer plusieurs souffrances chez les femmes qui vieillissent.

Être atteint d'une maladie létale comme un cancer incurable est aussi une expérience qui fait appel au temps. Un tel diagnostic rappelle que le temps est compté, que la vie aura une fin et que cette fin se rapproche. La mort qui se pointe marque la fin de la vie, ce qui implique souvent tout un processus de deuil et diverses souffrances psychologiques.

Dans une société vieillissante où les femmes ont une espérance de vie plus longue que les hommes, les femmes âgées sont nombreuses (Charpentier et al., 2010 ; Statistiques Canada, 2007). Or, les risques de cancer étant accrus par le vieillissement, plusieurs femmes âgées sont atteintes d'un cancer incurable (Statistiques Canada, 2007). Malgré leur nombre imposant, très peu de recherches s'attardent à comprendre leur expérience. Pourtant, être atteinte d'un cancer incurable est une expérience très souffrante à vivre, et ce, peu importe que I'on soit âgée ou non. Comme le soulevait Ionesco (1963): «on meurt tous pour la première fois. Que l'on soit jeune ou plus vieux, savoir que I'on a une maladie incurable reste une expérience limite, avec toutes les souffrances que cela implique». En dépit de cela, très peu de recherches donnent la parole aux aînés ou aux personnes atteintes de maladies incurables dans l'étude de leurs souffrances. Nous croyons que la compréhension de la souffrance que vivent les femmes âgées atteintes d'un cancer incurable commence par l'écoute de leur témoignage. C'est pourquoi nous avons choisi de donner la parole à ces femmes dans le cadre de cette étude. 


\section{La souffrance chez Paul Ricœur}

Cette recherche se fonde sur la conception de la souffrance élaborée par le philosophe Paul Ricœur. Celui-ci a présenté une réflexion sur la souffrance dès 1950, dans le premier tome de son livre Philosophie de la volonté. Dans ce texte, l'auteur soutient que la souffrance est une forme particulièrement vive de conscience de soi. Il relie cette réflexion sur la souffrance au vieillissement et à la mort. En effet, à l'image de la mort et du vieillissement, la souffrance resterait toujours l'étrangère (Ricœur, 1950). Ce texte est aussi I'occasion d'une réflexion sur le temps et la souffrance. En effet, Ricœur y traite de la tristesse de la contingence en faisant référence à la «négativité révélée par la souffrance» (Ricœur, 1950, p. 562), à cette négation du volontaire qui se révèle dans l'affectivité. Par rapport à la positivité de la croissance selon I'auteur, le temps est aussi menace et négativité. Il l'est d'abord en vertu de l'irréversibilité de la durée: le temps nous échappe, il va souvent sans se soucier de notre volonté. Le futur arrive inexorablement, selon son rythme propre et ce, tant dans le temps de l'impatience (l'attente) que dans le temps de la crainte (non, pas tout de suite), dira Ricœur (1950). De même, il ajoute que le passé ne peut être ni retenu, ni changé : à propos du passé, nous avons parfois à dire «cela été fait et ne peut être effacé» et «plus jamais je ne pourrai refaire cela, c'est terminé». Finalement, il ajoute que le présent révèle aussi le temps en tant que principe de dispersion de soi: le fait que chaque instant soit différent pose une épreuve à notre vœu d'unité dans l'existence, surtout lors d'une crise qui met en cause notre identité. L'auteur soutient aussi que la souffrance se donne comme le sentiment de notre diminution, concept qui sera au cœur de sa future élaboration de la souffrance. En effet, la réflexion de Ricœur sur la souffrance fut nourrie, circonscrite et précisée avec les années et fit l'objet d'un texte en 1994.

Dans ce texte, Ricœur distingue clairement la douleur de la souffrance, suggérant de «réserver le terme douleur à des affects ressentis comme localisés dans des organes particuliers du corps ou dans le corps tout entier» (Ricœur, 1994, p. 59). 
Ricœur soutient que la souffrance est «diminution de la puissance d'agir» (Ricœur, 1994, p.59) que I'on peut classer sur deux axes, tel qu'il le précise dans cet extrait:

Le premier est celui du rapport soi-autrui; comment, dans ces signes, le souffrir se donne conjointement comme altération du rapport à soi et du rapport à autrui. Le second axe est celui de l'agir-pâtir. (Ricœur, 1994, p.59).

Les signes de cette «diminution de la puissance d'agir» s'observent dans la parole, l'action, le récit et l'estime de soi. Abiven (1994, p. 206) résume bien la conception de Ricœur lorsqu'il affirme:

la souffrance est liée à la prise de conscience d'une limitation de soi vis-à-vis de soi-même, comme vis-à-vis d'autrui. Le noyau dur, le cœur de la souffrance a à voir avec la limitation et donc, une sorte d'impuissance. Selon cette conception, on pourrait alors la définir comme l'état douloureux d'une conscience réalisant que ce qu'elle vit, l'état dans lequel elle se trouve provoque limitation de son soi et impuissance.

Cette conceptualisation de la souffrance est très intéressante puisqu'elle définit, d'une manière très juste et bien élaborée, la nature de celle-ci. Elle propose aussi des signes qui permettent de la reconnaître. La réflexion de Ricœur sur la souffrance, le temps, le vieillissement et la mort est aussi particulièrement riche et pertinente à notre sujet d'étude.

\section{La souffrance et le temps}

Cette réflexion de Ricœur sur la souffrance et le temps nous a poussée à explorer les écrits qui portaient sur la souffrance, le temps, le vieillissement et le cancer incurable. Cela nous a permis d'identifier divers éléments qui pourraient être souffrants dans l'expérience du temps des femmes âgées atteintes d'un cancer incurable. En voici une synthèse.

\section{Raccordement des trois dimensions du temps}

La temporalité est depuis longtemps un sujet d'étude important en psychopathologie phénoménologique (Fuchs, 2001). Un champ d'études s'attarde au «temps vécu» et à ses impacts en psychopathologie. 
Minkowski (1933) notamment a développé ce domaine en étudiant les modifications de l'expérience du temps vécu et la psychopathologie. S'inspirant des travaux de Minkowski, Fuchs (2001) a développé le concept de désynchronisation. Il avance que si l'individu en santé mentale a l'impression de vivre en synchronie avec le temps d'autrui, l'individu atteint d'une psychopathologie se sent souvent déphasé (Fuchs, 2001, Kupke, 2005). Dans une société où I'on fait la promotion d'un temps accéléré, de logiques de productivité et de rentabilité (Des Aulniers, 1997; Thomas et al.,1992), ce qui s'associe à la décélération, comme le vieillissement, la maladie et la mort, est bien mal perçu et se module difficilement au temps accéléré (Lussier et al., 2006). Or, la sensation de ne pas être synchronisé avec le temps «social» serait un élément de souffrance importante (Fuchs, 2001).

Il arrive aussi que trois instances du temps (présent, passé et futur) ne soient plus raccordées, c'est-à-dire liées les unes aux autres (LanteriLaura, 1993). L'annonce du diagnostic de la maladie, l'expérience des traitements, notamment, provoqueraient souvent ce que Bury (1982) a appelé la «rupture biographique», une coupure dans le récit de la personne, dans la perception de soi et dans l'organisation de sa vie (Bury 1982, Charmaz 1983; Saaradon Eck, 2009). Cette rupture dans le récit de la personne pourrait nuire au raccordement des temps

Des Aulniers (1997) a étudié les facteurs de cohérence entre les pratiques visant à composer avec la maladie grave et la représentation du temps. Elle a alors démontré que les éléments suivants sont liés à une incohérence: un rapport tronqué au passé (un passé qui serait hypertrophié, idéalisé ou simplement rayé de la conscience), une «élémentalité auto-injectée» (le fait de vivre au jour le jour) et I'incertitude importante face au futur. Ces facteurs qui nuisent à la cohérence semblent se lier à un manque de raccordement des trois temps et être source de souffrances, altérant le rapport à soi, à son histoire.

\section{La souffrance et le passé}

La souffrance des femmes âgées atteintes de cancer peut également se rattacher à leurs représentations de leur passé. Cela se rapporte à la réminiscence, terme utilisé pour expliquer comment les aînés construisent socialement le sens accordé à leur vie (Covan, 2005). En 
effet, I'histoire de chacun et le sens que I'on arrive ou non à y accorder peuvent être source de souffrances. Parfois, face à la maladie grave, I'individu entreprend une quête de sens. Or, pour l'aîné qui n'arrive pas à donner sens à son histoire, on devine qu'une expérience de souffrance importante peut alors surgir. Selon Wyllie (2005), certains considèrent leur passé comme un «passé mort» (dead past), un passé cristallisé auquel ils n'arrivent pas à donner sens. Selon lui, pour donner sens au passé, il faut avoir un futur ouvert. Sans futur ouvert, il n'est pas possible de réinterpréter le passé, de le «vitaliser» pourrait-on dire, et d'éviter que ce dernier ne devienne un «passé mort». On revient ici encore une fois à l'importance de raccorder les trois modalités du temps.

\section{- La souffrance et le présent}

\section{La souffrance comme arrêt, éternel présent}

L'expérience de la souffrance est aussi liée à l'expérience du présent. Comme le rappelle Thomas (2004) souffrir est passif, nous dépossède. La souffrance modifie l'expérience du temps vécu en donnant l'impression que le présent est «éternel», que le temps s'est soudainement arrêté (Wyllie, 2005). Ce sentiment d'immobilité est parfois tellement étouffant que l'individu sombre dans le désespoir (Minkowski, 1933).

\section{Le patient impatient ...}

Rappelons aussi que dès son entrée dans le monde médical, la personne atteinte de cancer se voit appelée «patiente», un terme qui fait appel à la notion de temps (Froucht-Hirsch, 2005), d'attente. Or le patient peut parfois vivre de l'impatience (Froucht-Hirsch, 2005). Certains vont refuser le présent souvent inassimilable et désirer se défiler devant la dureté de ce dernier. Pour un certain temps, certains chercheront à se réfugier dans un futur ou un passé dans lequel cette menace de mort est absente (Des Aulniers, 2009; Kuupplelomäki et al., 1998). Puis, les gens adopteront diverses positions face au temps, certains souhaiteront redevenir comme avant (donc dans le passé) alors que d'autres voudront cheminer vers un état qui ne sera jamais comme avant ou apprendre de cette épreuve (Froucht-Hirsch, 2005). Quelques-uns regretteront des actions posées dans le passé qui, selon 
eux, pourraient avoir causé leur maladie, d'autres regretteront tout simplement un passé sans maladie, etc. Certains souhaiteront qu'un futur plus heureux émerge au plus vite alors que d'autres auront une peur persistante du futur.

\section{La souffrance et le futur}

\section{Le désespoir}

Il arrive aussi que l'avenir soit considéré comme «à-venir» (Des Aulniers, 1997, p. 12). Cela peut arriver à la personne âgée qui considère ne plus avoir droit à l'espoir car tout est joué et que le futur n'est que la mort. Minkowski (1933) dira que le désespoir donne l'impression que l'avenir est fermé. Or, vivre cette impression que le futur est bloqué mène souvent au désespoir.

\section{L'incertitude et la mort}

Face au futur, les personnes atteintes de cancer vivent aussi beaucoup d'incertitude (Kuupelomäki et al., 1998). Or, l'incertitude est souvent une expérience très souffrante, directement liée à l'impuissance. M'Uzan (1977) rapporte que la personne atteinte d'une maladie mortelle est consciente qu'elle dispose de peu de temps pour accomplir sa tâche de deuil (deuil de sa propre vie, de son entourage, etc.). Son présent prend alors une très grande valeur alors que le futur prend le visage de la mort, et ce, de manière beaucoup plus concrète qu'à I'habitude. Or, faire face à la mort est une épreuve qui peut être très souffrante. Confronté à cette réalité, le futur peut rester ouvert, malgré la mort, ou être bloqué (et alors témoigner d'un certain désespoir).

\section{LES QUESTIONNEMENTS QUI ONT ANIMÉ CETTE RECHERCHE}

Malgré ces nombreux indices qui laissent croire que les représentations du temps peuvent se lier à l'expérience de la souffrance chez les femmes âgées atteintes d'un cancer incurable, il reste qu'à notre connaissance, leur représentation du temps n'est pas explorée dans la 
recherche. Ainsi, I'analyse de l'expérience du temps vécu peut contribuer à mieux comprendre et redéfinir les contours de leur souffrance. Comment la souffrance des femmes âgées atteintes d'un cancer incurable peut-elle se lier à leur interprétation du temps? Comment se lie-t-elle à leur interprétation de leur passé? Comment se lie-t-elle à leur interprétation du présent? Comment se lie-t-elle à leur interprétation du futur? Comment cette souffrance se lie-t-elle à leur manière de raccorder les trois instances du temps? L'expérience de la maladie grave affecte-t-elle le «raccordement» entre les trois dimensions du temps, et alors être vécue comme expérience de «désynchronisation »? Poser de telles questions permet de mieux comprendre si et comment la représentation du présent, du passé et du futur peut être source de souffrances chez les femmes de cette population.

\section{Méthodologie}

Pour répondre à ces questions nous avons réalisé une étude qualitative exploratoire issue du courant de la psychologie humaniste existentialiste. La recherche qualitative est particulièrement pertinente à l'étude de la souffrance. Ce type de recherche et les choix méthodologiques qui l'accompagnent ici laissent aussi place à l'expression de la parole de la personne sur sa souffrance, un acte qui en soi lui redonne le pouvoir de dire, ce qui rejoint la conception de la souffrance relative à I'impuissance à dire (Ricœur, 1994). Cette recherche est tirée du projet: «La souffrance chez les femmes âgées atteintes d'un cancer incurable», financée par l'agence de la santé publique du Canada et parrainée par le CSSS Cavendish.

\section{Recrutement}

Le recrutement des participantes s'est fait au sein du Centre hospitalier de I'Université de Montréal (CHUM), dans les unités de soins spécialisés en cancer colorectal, en cancer du poumon et en cancer hépatobiliaire, ainsi qu'au Centre de santé et de services sociaux Cavendish, tous situés à Montréal. L'échantillon fut constitué selon la méthode non probabiliste d'échantillon typique (Mayer et al., 2000). Ainsi, nous avons cherché à rencontrer des femmes atteintes de divers cancers, issues de 
divers milieux socio-économiques et d'âges différents. Les critères d'inclusion des participantes de notre recherche étaient les suivants: être une femme âgée de 65 ans et plus, parler français ou anglais, avoir reçu un diagnostic de cancer incurable et connaître ce diagnostic. Nous avons recruté 10 participantes. L'arrêt du recrutement a été motivé par la richesse et l'ampleur des discours livrés lors des entrevues, la diversité des témoignages, des caractéristiques des participantes et des problématiques soulevées. De plus, nous avions réussi à avoir une certaine diversité dans notre échantillon (différents âges, milieux socio-économiques, types de cancer), tel que nous le souhaitions.

\section{Entretiens}

Des entrevues semi-dirigées ont été réalisées avec les participantes. Elles ont été effectuées par la chercheure principale de cette recherche, expérimentée à réaliser ce type d'entretien. Un canevas d'entrevues formé de questions ouvertes qui abordent les thématiques de cette étude a été utilisé dans le cadre de ces entretiens. Ces questions portent plus précisément sur la conception de la souffrance des femmes rencontrées, les liens qui pourraient unir leurs souffrances actuelles et leur passé, leur présent et leur futur. Des questions étaient aussi posées sur la place que prend le passé, présent et futur dans leur vie actuelle.

Puisque les sujets abordés étaient délicats, les thèmes furent abordés graduellement. Les questions ont été posées en respectant le plus possible le rythme, la manière de se mettre en récit, les désirs et réticences de chacune des participantes.

Il est à noter que puisque les femmes rencontrées étaient malades la durée de l'entrevue était variable, toujours déterminée par le désir de I'interviewée de poursuivre et par sa capacité physique à le faire. Les entrevues ont, par conséquent, duré de 40 minutes à 3 heures. Les entrevues qui ont été plus longues l'ont été car les participantes souhaitaient continuer à parler. Une $(n=1)$ à deux $(n=9)$ entrevues ont été menées avec chaque participante afin d'aborder tous les thèmes que nous désirions étudier. Avec une participante, un seul entretien a permis de couvrir tous les thèmes, tandis qu'avec les autres répondantes deux entretiens ont permis d'aborder les différentes thématiques. 


\section{Analyse}

À partir des témoignages recueillis, une analyse de catégories conceptualisantes des données fut réalisée (Paillé et al., 2012). Les entrevues ont été transcrites et relues à plusieurs reprises. Une liste de thèmes et de rubriques fut construite à partir du contenu des premières entrevues, et permis de construire l'arbre thématique. Cet arbre thématique fut révisé au fur et à mesure que les autres entrevues ont été codées. Toutes les entrevues furent ensuite codées avec la liste de thèmes et les rubriques furent révisées. Nous avons ensuite relu le contenu verbatim de chaque thème et rubrique pour en construire une synthèse et structurer le contenu analysé. Nous avons alors regroupé les rubriques qui se ressemblaient, se reliaient, s'éclairaient, en ordonnant les thèmes de manière à mettre en lumière leur articulation, leurs nuances, points communs, etc. Nous avons ensuite cherché à aller un peu plus loin dans l'analyse, dynamisé cette dernière et interprété davantage les données en créant des catégories conceptualisantes (Paillé et Mucchielli, 2012). Pour ce faire, nous avons formulé des catégories qui nous permettraient de répondre à nos questions de recherche.

La validité de l'analyse fut assurée en clarifiant l'appareil théorique, enregistrant les entretiens et valorisant des concepts proches de l'expérience vécue des participantes (Des Aulniers, 1993). Une comparaison interjuge fut également réalisée en faisant codifier chaque entrevue par deux personnes pour ensuite comparer les codifications. Les règles d'exhaustivité, de représentativité, d'homogénéité, de pertinence et d'univocité furent aussi respectées dans la catégorisation et lors des analyses (Mayer et al., 2000).

\section{Résultats: La souffrance et le temps}

Les résultats de cette étude sont présentés en quatre temps: 1- la section «passé» porte sur les événements du passé qui se lient aux souffrances des participantes, 2- la section «présent» sur leurs manières d'interpréter le présent en lien avec leurs souffrances et 3- la section «futur» aborde les souffrances liées à leur vision du futur. L'analyse se conclut sur 4- une section portant sur le rythme du temps vécu par les participantes. 
La souffrance que la majorité des répondantes ont abordée lorsqu'elles parlaient de leur passé se lie à I'histoire de leur maladie $(n=8)^{1}$ et plus précisément à deux événements de leur passé: I'attente et l'annonce du diagnostic du cancer.

\section{La souffrance de l'attente}

La moitié des participantes rapportent que l'attente qu'elles ont vécue entre le moment où elles-mêmes et/ou les professionnels de la santé ont eu l'intuition qu'elles étaient malades et le moment où elles ont obtenu le diagnostic de leur cancer fut très souffrante à vivre $(n=5)$. Elles ont alors été placées dans une position précaire d'impuissance qui rejoint la conception de Ricœur de la souffrance de l'attente (1950) et de la souffrance comme impuissance à faire (par exemple à tenter de se guérir), impuissance à dire (par exemple à nommer leur maladie), à comprendre ce qui leur arrive (par exemple, savoir ce qu'elles ont) et ce qui les attend (Ricœur, 1994). Ainsi, une participante raconte que ce qui est souffrant à vivre dans son expérience d'avoir un cancer: «c'est qu'ils te donnent pas de traitement, ils font rien. Ils font rien que des tests, puis des tests, puis des tests. Puis ça prend une éternité $[\ldots] \gg\left(10,1^{2}\right)$

Le temps d'attente qui sépare le moment du diagnostic des traitements est un événement qui fut souvent très dur à vivre pour les participantes. Ce laps de temps qui s'est écoulé est interprété par certaines comme étant directement lié à l'aggravation de leur maladie, voire à sa létalité $(n=3)$. Les souvenirs de ces moments passés à attendre, impuissantes devant la maladie qui progressait, étaient chargés de plusieurs émotions telles que l'angoisse, la colère, la tristesse et le regret. Une fois de plus ces femmes ont alors fait face à une impuissance atteignant leur pouvoir faire, ce que I'on pourrait classer sur l'axe agir-pâtir de la conception de la souffrance de Ricœur (1994).

\section{L'annonce du diagnostic: une brèche dans son histoire}

Lorsqu'elles parlent de leur passé, plusieurs participantes racontent aussi comment l'annonce de leur cancer fut difficile $(n=7)$. Même si I'annonce vient cesser l'attente, le diagnostic brise aussi parfois l'espoir des participantes (de ne pas avoir le cancer, de guérison, etc.). Celles-ci 
décrivent comment elles ont été «secouées» par la nouvelle $(n=1)$, comme elles ont eu de la difficulté à y croire $(n=2)$. Dans certains cas, la manière d'annoncer le cancer (au téléphone ou encore de manière peu empathique) fut source de souffrances $(n=3)$ que l'on peut relier à I'axe soi-autrui de Ricœur (1994). L'événement de l'annonce du cancer semble souvent difficile à intégrer à I'histoire de ces femmes, ce qui rappelle les propos de Ricœur sur le temps comme facteur de la dispersion de soi dans la crise qui menace notre souhait d'unité dans l'existence (1950). L'annonce marque une coupure dans le temps: avant de savoir qu'elles avaient un cancer et après. C'est un événement saillant de leur passé lourd de conséquences pour le futur.

\section{Deuxième temps : la souffrance et le présent}

L'expérience du présent de ces femmes est aussi teintée de maintes souffrances.

\section{Vivre au jour le jour pour composer avec l'impuissance}

La grande majorité des participantes soutiennent qu'elles adhèrent à l'idée de vivre au jour le jour $(n=8)$. Cela les aide parfois à composer avec la souffrance liée aux difficultés du quotidien, aux angoisses face à la mort et à l'incertitude et peut ainsi être considéré comme une stratégie d'adaptation. Une participante rapporte ce qui l'aide:

C'est de vivre ce que tu es capable de vivre chaque jour, sinon si tu t'apitoies sur ton sort puis tu te dis ah... je suis malade, je fais plus ci, je fais plus ça, dans ce temps-là tu penses juste à aller te coucher puis à pas te relever. $(3,1)$.

C'est pour éviter de souffrir davantage face aux pertes qu'elle vit (impuissance à faire des choses qu'elle faisait autrefois et donc qui affecte le pouvoir faire de l'axe agir-pâtir de Ricœur (1994)) et de sombrer dans le désespoir (voire le suicide auquel elle pourrait référer dans l'idée de ne pas se relever?) que cette dernière décide de vivre au jour le jour.

Cinq participantes disent vivre au jour le jour car leur futur est imprévisible, incertain $(n=5)$. Une d'elles dit que c'est parce qu'elle ne croit pas qu'elle peut faire de projets pour le futur qu'elle se centre sur son 
présent $(3,1)$. Une autre aborde son incertitude en parlant de la précarité de sa santé et du fait qu'elle peut mourir subitement à tout moment $(8,1)$. Ne sachant pas ce que le futur lui réserve et tentant de ne pas avoir peur de la mort, elle décide de se centrer sur le moment présent pour composer avec cette réalité, ce qui peut également être considéré comme étant une stratégie adaptative.

Deux de ces participantes qui disent vivre au jour le jour ajoutent que cette imprévisibilité de leur maladie est telle qu'elles sentent qu'elles n'ont pas le choix de vivre au jour le jour, que c'est une nécessité. Devant cette impuissance face à leur futur et pour s'en protéger, elles décident de le garder hors de leur champ de conscience en se centrant sur le présent. Certaines disent effectivement vivre au jour le jour parce qu'elles refusent de penser au futur $(n=3)$. En voici un exemple:

Le futur? Ça ne me dérange pas, je me dis que si ça arrive, ça arrive. C'est mieux de même, je le prends. C'est mieux de même parce qu'on deviendrait fou si y on pensait tout le temps. C'est vrai. Non, je ne pense pas à...Ça va jour par jour. $(1,1)$

D'autres participantes disent qu'elles vivent au jour le jour parce qu'elles ne peuvent plus faire de projets $(n=3)$. Il y a toutefois des revers au fait de vivre au jour le jour sans faire de projets, ainsi une participante $(8,1)$ raconte que souvent, lorsqu'elle va un peu mieux, elle n'a rien à faire parce qu'elle ne fait plus de projets. Dans ce cas, le fait de ne vivre qu'au moment présent, sans se projeter dans le futur fait que cette dernière se retrouve devant le vide lorsque ce futur devient présent.

\section{Profiter du moment présent en réponse à l'incertitude}

Certaines participantes disent apprécier davantage les «petites» joies du quotidien depuis qu'elles savent qu'elles ont le cancer $(n=3)$. Une d'elles relie cela à son impuissance:

On prend les bons moments qui passent et puis [...] je ne peux pas faire mieux. Quand bien même que je me révolterais, quand bien même je crierais, quand bien même que je m'en prendrais à la balance du monde, ça va changer quoi? La maladie sera toujours là, je ne serai pas plus guérie, alors... C'est à moi que je fais mal, c'est aussi bien de prendre les bons moments qui 
passent, de les apprécier, parce que il va y avoir des moments qui vont être moins bons [...]. Et puis il arrivera ce qui arrivera plus tard. $(3,1)$

Elle soulève ici comment le fait d'avoir une attitude de révolte ne changerait rien à sa situation et qu'elle préfère donc tenter de profiter des joies qui passent. Elle adopte aussi cette attitude parce qu'elle sent que des moments plus difficiles arriveront.

La mort qui se pointe à I'horizon marque cette manière qu'ont les participantes de vivre le moment présent comme en témoigne cette participante: «J'essaye de vivre comme si...Pendant que je suis bien là, je vais en profiter. C'est ça. Quand je pourrai plus bien là, ce sera autre chose. 》 $(10,2)$

Il semble que c'est parce qu'elles savent que le temps est limité par la mort qui s'approche et qu'elles sont impuissantes devant cet inévitable que ces femmes apprécient ces moments de leur présent et en profitent au maximum. Plutôt que de se centrer sur leurs limites, elles se centrent sur les capacités qu'elles possèdent toujours afin de composer avec la souffrance de l'impuissance (Ricœur, 1994). Devant ce passé révolu, ce futur incertain qui leur échappe et qui est source de souffrance (Ricœur, 1950) et face à la constatation d'une diminution du pouvoir faire (de l'axe agir-pâtir de Ricœur (1994)), le présent devient l'ultime refuge de la volonté de ces femmes.

\section{Troisième temps : la souffrance et le futur}

On constate que l'incertitude et les craintes face au futur teintent le rapport au présent des participantes. Ici, le présent et le futur se rejoignent clairement. Outre l'incertitude abordée, la manière d'interpréter le futur se lie aussi aux souffrances de ces femmes à différents niveaux.

Certaines racontent qu'elles ont l'impression de ne pas avoir de futur, de ne plus pouvoir faire de projets $(n=2)$. Cette impression est une manifestation de souffrance atteignant directement le pouvoir faire, notamment, de l'axe agir-pâtir décrit par Ricœur (1994), une manifestation de désespoir. 


\section{Réflexions sur la mort et le mourir}

Toutes les participantes ont parlé de la mort à un moment ou l'autre des entrevues, même si aucune question ne portait directement sur cette thématique $(n=10)$. Ainsi, la mort fait partie de leur horizon, de leur réflexion sur le temps et que ce soit pour nous dire qu'elles veulent y penser ou non, elle était présente dans leur discours.

Les réflexions et manières d'envisager la mort des participantes sont très différentes les unes des autres dans leur nature et dans leur manière de se lier à leurs souffrances. Cinq participantes disent penser à la mort lorsqu'elles réfléchissent à leur futur $(n=5)$. Une d'elles dit au sujet de son futur: «Bien d'habitude je vois la mort. Je me dis je peux pas toffer [durer, survivre] de même.» $(9,2)$. Le futur, pour elle, c'est la mort.

Deux participantes rencontrées ont une vision très optimiste de la mort et elle n'est pas envisagée comme quelque chose de souffrant, ce qu'elles relient au fait qu'elles ont vécu des expériences où elles ont frôlé la mort $(n=2)$. Une d'elles parle de son expérience de mort clinique disant qu'elle était bien et qu'elle aurait préféré ne pas être ranimée $(7,1)$. Elle ajoute que depuis elle n'a plus peur de la mort, que la mort est la fin des soucis. Une autre raconte que depuis qu'elle a vécu une expérience similaire, elle n'a plus peur de la mort. Ainsi, la mort n'est pas pour elles une source de peurs. Au contraire, comme elle le rajoute:

j'étais tellement bien que je me suis dit la mort c'est ça pour moi c'est quelque chose de bien. C'est un réconfort. Fait que...depuis ce temps-là la mort moi là je me dis en moi-même c'est comment je te dirais ça... la récompense de ta vie. $(3,2)$.

La mort qui s'approche est ici source de réconfort, point final des souffrances de ce monde.

La moitié des participantes, en parlant de la mort, nous disent toutefois qu'elles ne se sentent pas prêtes à mourir $(n=5)$. Une d'elles explique qu'elle chérit l'espoir de vivre plus longtemps, pour pouvoir accomplir certains projets $(5,2)$. Une autre rapporte toutefois qu'à l'inverse si sa santé reste comme elle est actuellement, elle préfèrerait mourir $(4,2)$. La mort est vue par elle comme une libération. D'autres disent qu'elles 
sont prêtes à mourir $(n=2)$ ou qu'elles n'ont pas peur de la mort $(n=3)$ parce qu'elle est cessation des douleurs. Toutefois, il est à noter que d'une entrevue à l'autre, il arrive que cette conception de la mort change, tel qu'on le constate notamment avec une participante qui dit ne pas avoir peur de la mort lors de la première entrevue puis nous confie lors de la seconde entrevue que sa vision a changé et qu'elle a très peur de mourir. On voit ici que la peur de la mort est variable, elle peut être plus ou moins forte, présente ou absente, d'un moment à I'autre chez une même personne.

\section{Le futur et l'espérance de vie}

Les participantes abordent aussi une dimension de leur futur qui se relie à leurs souffrances lorsqu'elles parlent de leur cancer: leur espérance de vie. Quelques-unes se questionnent face à leur futur, veulent savoir quelle est leur espérance de vie $(n=3)$. Pour plusieurs, il n'est pas possible d'avoir de réponses aux questions au sujet de leur espérance de vie, et cette donnée reste incertaine. L'espérance de vie est interprétée différemment par les participantes, mais les prévisions et/ ou les réponses à ces questions sont souvent source de souffrances.

Certaines espèrent survivre au-delà de cette prévision des professionnels de la santé $(n=2)$. Une participante raconte qu'elle espère que les durs traitements qu'elle a subis ne l'ont pas été en vain. Chez elle, la notion d'espérance de vie porte aussi une part d'espoir: espoir que la vie se prolonge au-delà de cette prédiction, qu'elle l'atteigne, espoir de durée, espoir de vivre.

Toutefois, comme l'ajoute une autre, tout n'est pas dans la durée de la vie:

Je me dis il faut que je me repose, il faut que je me force pour guérir puis on me dit que je guérirai jamais, fais que je me dis pourquoi est-ce que je me force autant? Je me force à me reposer pour que je dure plus longtemps. Si je me repose pas assez je vais venir plus fatiguée, je vais venir plus malade et je vais mourir encore plus vite. [...] Mais est-ce que durer plus longtemps, en faisant des affaires plates, c'est mieux que de faire des tas de choses le fun, mais durer moins longtemps? Là je suis en vrai dilemme. $(8,1)$ 
Ici, la contradiction qui existe entre le fait de tenter de guérir (lorsque la participante dit «il faut que je me force pour guérir») et le caractère incurable de la maladie se fait sentir, même si cette participante semble bien consciente que cette mort qui approche est inévitable... Cette participante relève aussi la question de la durée, versus la qualité de vie. Son témoignage pose la question: est-ce mieux de vivre plus longtemps ou plus heureux? Une autre femme rencontrée, dans un même ordre d'idées, rapporte qu'elle refuse de vivre des traitements qui pourraient allonger son espérance de vie parce que:

Les traitements ont été durs [...] Je trouve qu'on est pris pour des cobayes. Là je vais leur dire si ça va pas je mourrai, là. Ils ont dit: vous pourriez vivre 2-3 ans avec un autre traitement. Oui mais tu pâtis! $(4,1)$

Elle refuse de vivre longtemps en souffrant, préférant mourir que d'avoir à subir à nouveau de tels traitements.

\section{Le rythme du temps vécu: un ralentissement qui dérange, un arrêt refusé}

Les participantes nous ont aussi parlé du ralentissement auquel elles font face. Quatre disent qu'elles ont l'impression d'être ralenties depuis qu'elles ont le cancer et qu'elles sont plus âgées. Elles ne vivent plus au même rythme qu'avant. Ce ralentissement en irrite quelques-unes $(n=3)$. Une raconte que ce ralentissement est difficile à vivre $(5,1)$, et une seconde qu'elle en est «tannée» $(7,1)$. Ce ralentissement se lie aussi aux pertes d'autonomies que vivent plusieurs et qui se relie à leurs souffrances. Il semble ici que ce soit le ralentissement par rapport à leur propre vitesse antérieure qui dérange ces participantes. Les limitations au niveau de la vitesse d'exécution des tâches atteignant ici le pouvoir faire de l'axe agir-pâtir de Ricœur (1994). En effet, la longueur du temps passé à faire des choses autrefois beaucoup plus rapides limite la quantité et la nature de ce qui peut être accompli.

Certaines participantes, malgré ou face à ce ralentissement qu'elles observent, racontent qu'il est important pour elles de ne pas s'arrêter $(n=2)$. Une d'elles affirme:

C'est juste ça, continuer, ne jamais arrêter, puis tu te revires pas de bord pour regarder, oui tu regardes ce que tu as fait mais tu continues. Faut pas que tu arrêtes, si tu arrêtes tu es finie. $(1,1)$ 
Le fait d'arrêter de penser à sa mort, où sa souffrance semble ici perçu comme quelque chose de trop difficile à vivre (comme on peut le voir dans l'expression: «si tu arrêtes tu es finie»). On peut ici établir un parallèle entre le fait de s'arrêter et de mourir. Une autre raconte comme elle se force à avancer, toujours pour «revenir» $(7,1)$. On voit que sous cette volonté d'avancer, de ne pas arrêter, il y a l'espoir d'aller mieux, mais sous l'expression «revenir» on peut aussi se demander s'il n'y a pas une fois de plus, une référence à la mort (comme dans l'expression revenir à la vie par exemple).

\section{Réflexion sur ces résultats}

\section{Souffrances liées au passé: l'attente et l'annonce du diagnostic}

Cette étude permet de constater que les liens qui unissent la souffrance des femmes âgées atteintes de cancer incurable et leur interprétation du temps sont nombreux. L'attente du diagnostic et du traitement sont des moments du passé des femmes rencontrées qui furent particulièrement souffrants à vivre. L'attente les a fortement ébranlées, les plaçant dans une position d'impuissance très souffrante qui rappelle celle décrite par Ricœur (1950, 1994). L'annonce du cancer est aussi un événement souffrant du passé qui marque une brèche dans I'histoire de ces femmes qui arrivent parfois difficilement à l'intégrer à leur récit. Cette annonce marque l'arrivée d'un nouveau marqueur identitaire: celui de personne atteinte de cancer, ce qui révèle le temps comme facteur de dispersion de soi (Ricœur 1950) et, dans la crise, comme source de souffrance. Cette difficulté d'intégrer cet événement à leur histoire, rend effectivement difficile le raccordement les trois instances du temps (passé, présent, futur) (Lanteri-Laura, 1993). Enfin, il est aussi possible que le fait que l'événement revête certaines caractéristiques du trauma (notamment la menace de mort) le rende particulièrement difficile à intégrer à son histoire, à l'image du trauma. 
Souffrances liées au présent: se centrer

sur le moment présent face à la mort et l'incertitude

L'interprétation du présent des femmes rencontrées témoigne aussi de grandes souffrances. Plusieurs disent vivre au jour le jour, être axées sur le moment présent, parce qu'elles ne veulent pas penser au futur, un futur souffrant, source d'impuissance, d'incertitudes, de peurs, d'angoisses. Ce «temps amputé» (Des Aulniers, 2009) qui n'est pas raccordé au futur semble être un refuge plus ou moins efficace contre les angoisses face au futur. Dans certains cas, il peut s'inscrire dans un processus d'adaptation à une situation difficile. Dans la mesure où cela est un choix, une action, ce n'est pas une impuissance telle que définie par Ricœur (1994) mais revêt plutôt les traits d'une stratégie d'adaptation. Toutefois, certaines disent ne pas avoir le choix de vivre au jour le jour, parce que le futur est trop difficile à envisager, ce qui rappelle la négativité associée à l'irréversibilité du temps par Ricœur (1950). Vivre au jour le jour signifie parfois ne pas penser au futur, ce qui traduit une difficulté à raccorder les trois instances du temps (Lanteri-Laura, 1993). On voit aussi derrière cette centration sur le présent, l'ombre du désespoir de certaines participantes qui n'arrivent pas à se projeter dans le futur, considèrent que leur futur est bloqué, ce qui rejoint la définition du désespoir (Willie, 2005). Cette impuissance face au futur des femmes rencontrées rappelle aussi l'analyse de la souffrance comme impuissance de Ricœur (1950, 1994), une fois de plus.

Ces femmes valorisent aussi beaucoup les capacités dont elles disposent actuellement, appréciant chaque moment qui passe, bien conscientes de la valeur de ces moments limités par la mort qui s'approche ce qui rappelle ce que soulevait M'Uzan (1977). C'est une fois de plus en réaction à l'impuissance face à ce qui les attend qu'elles adoptent cette attitude (Ricœur, 1950, 1994).

Souffrances liées au futur: l'espoir, le désespoir... et la mort

Le futur est pour certaines bloqué, fini. Cela est évidemment une conception du futur très souffrante, affectant directement le pouvoir faire de la personne (Ricœur, 1994). Le sentiment de ne plus pouvoir faire de projets, de ne pas avoir de futur est aussi une manifestation de désespoir (Willie, 2005; Minkowski, 1933). 
La souffrance des participantes se lie souvent à leur vision ou réflexion sur la mort. Une participante se prépare à la mort, comme pour contrer la diminution de sa puissance d'agir (Ricœur, 1994) inhérente à la mort qui approche, alors que d'autres refusent d'y penser. Les interprétations de la mort diffèrent beaucoup d'une personne à l'autre et conséquemment elles se lient aussi différemment à la souffrance. Cela n'est pas surprenant, la tâche d'apprendre à mourir étant une tâche particulièrement intime, ardue, jamais finie, complexe et non linéaire.

\section{Un ralentissement qui dérange, un arrêt refusé}

Enfin, des participantes remarquent que leur rythme a ralenti avec la maladie. Elles n'apprécient pas les pertes qui viennent avec ce ralentissement. Il semble que ce ne soit pas ici la désynchronisation qui dérange ces femmes (et ainsi ne rejoint pas l'idée de Fuchs, 2001); leur souffrance est plutôt issue des pertes relatives à ce ralentissement. Cette souffrance se lie aux limitations de leur pouvoir faire (Ricœur, 1994). Certaines de ces participantes qui remarquent qu'elles ralentissent ajoutent qu'elles ne souhaitent pas arrêter, qu'elles se poussent à toujours avancer. L'arrêt dans ce cas est lié à la fin, il rappelle la mort et le puissant désir de vivre de ces femmes. Cette volonté d'avancer rappelle aussi I'héroïsme et l'attitude combative face à la maladie tellement valorisés dans notre société (Ben Soussan, 2004).

\section{Refonte d'une définition de la souffrance}

À partir de ces résultats, nous pouvons reformuler une définition de la souffrance fondée sur celle de Ricœur (1994) mais qui s'appliquerait plus particulièrement à la réalité des femmes âgées atteintes de cancer incurable et aussi, possiblement, à d'autres personnes. Ainsi, nous pourrions avancer que: la souffrance est le sentiment d'impuissance vécu lorsque la menace ou l'expérience d'un événement mortifère engendre la rupture d'un lien. En effet, il ressort de cette étude que le diagnostic de cancer incurable prend rapidement les traits de la mort, et qu'il est ce que l'on pourrait appeler une menace d'«événement mortifère». Ce que nous appelons ici «événement mortifère» causerait une forme de mort, de destruction qui peut bien évidemment être symbolique, sociale, mais aussi physique. À l'instar de la séparation dans I'axe soi-autrui dont Ricœur (1994) parlait, cet événement vient à notre avis inscrire la «rupture d'un lien» chez les participantes. Cette rupture 
peut être celle d'un lien de soi face à autrui ou face à soi-même. Face à autrui, cette rupture de lien peut prendre la forme d'une rupture de liens sociaux ou de relations. Face à soi-même, cela peut être une rupture dans la ligne du temps, soit dans le passé, le raccordement des trois instances du temps, dans l'interprétation du futur et l'expérience de son présent. L'impuissance, enfin, renvoie à l'affect de la souffrance, à son éprouvé, tel que nous l'avons constaté tout au long de cette recherche.

\section{Mieux comprendre la souffrance en clinique et en recherche}

Cette reformulation de la définition de la souffrance permet d'en comprendre les rouages complexes chez les femmes que nous avons rencontrées, tout en pouvant aussi s'appliquer à d'autres personnes. L'idée de rupture du lien rejoint l'idée de séparation de Ricœur (1994), toutefois nous préférons ici le terme rupture qui nous semble mieux nommer ce que les participantes vivent, l'idée de cassure et de dureté de cette division, notamment au niveau de la ligne du temps, de leur histoire. Le concept de rupture rejoint aussi celui de la «rupture biographique» vécue lors d'un diagnostic de maladie grave qui avait été soulevé par divers auteurs (Bury 1982; Charmaz, 1983; Saaradon Eck, 2009). Cette recherche confirme que plusieurs femmes âgées atteintes de cancer incurable expérimentent cette rupture biographique tout en élargissant l'expérience de la rupture à diverses formes de ruptures qui peuvent survenir (face à soi et face à autrui). L'introduction d'un événement mortifère qui engendre cette impuissance dont parle Ricœur (1994) permet aussi d'élargir sa définition, d'y introduire la dimension de la mort et sa relation avec la souffrance.

Au niveau clinique, nous avons aussi constaté que parler du temps avec des femmes atteintes d'un cancer incurable contribue à ce qu'elles se mettent en récit, raccordant un tant soit peu les trois instances du temps. Parler du futur ouvre la porte à une discussion sur la mort, si elles désirent en parler. Or, il est souvent difficile d'aborder la mort en recherche et en clinique, vu la délicatesse du sujet. Ce constat pourrait servir aux chercheurs et aux cliniciens qui interviennent auprès de personnes atteintes de maladies incurables. Cela permet également de mieux voir si ces personnes considèrent que leur futur est ouvert ou 
fermé, de deviner la présence de désespoir, ce qui gagne à être travaillé en clinique. Parler du passé met aussi parfois en lumière des événements difficiles, voire traumatiques, que ces personnes peinent à intégrer à leur histoire. Aborder le présent, l'attente, l'incertitude, permet de constater ce qui est souffrant à vivre dans leur expérience, ici et maintenant. Or, laisser la personne se raconter et entrer en dialogue avec elle, l'encourager à faire le récit de son histoire est en soi un processus qui invite à inscrire son histoire dans le temps, la raconter, la réviser, la comprendre, la reconstruire... C'est un travail de mémoire et de mise en récit qui n'est pas sans rappeler l'œuvre de Ricœur (2000), qui a soulevé avec tant de doigté l'importance de ce travail. C'est d'ailleurs aussi peut-être dans une idée de durée, que ces femmes ont accepté de participer à une recherche dont les résultats seront publiés après le décès de certaines, comme si elles voulaient inscrire leur histoire et expérience dans un temps qui les dépasserait.

\section{BIBLIOGRAPHIE}

ABIVEN, M. (1994). «Au bout du chemin», dans J.M. VON KAENEL et B. AJCHBAUM-BOFFETY (dir.), Souffrances, corps et âme, épreuves partagées, Paris, Éditions Autrement, p. 203-211.

BERSAY, C. (2004). «Vieillir», Études sur la mort, vol. 2, n 126, p. 37-42.

BOURGEOIS-GUÉRIN, V. (2010). «La souffrance en lien avec les écueils de la communication chez les femmes âgées atteintes de cancer incurable», Journal canadien du vieillissement, vol. 29, $\mathrm{n}^{\circ} 4$, p. 529-541.

BURY, M. (1982). «Chronic illness as biographical disruption», Sociology of Health and Illness, vol. 4, n², p. 167-182.

CHARMAZ, K. (1983). «Loss of self: a fundamental form of suffering in the chronically ill », Sociology of Health and Illness », vol. 5, p. 168-195.

CHARPENTIER, M., N. GUBERMAN, V. BILLETTE, J.-P. LAVOIE, A. GRENIER et I. OLAZABAL (2010). Vieillir au pluriel. Perspectives sociales, Québec, Presses de I'Université du Québec.

COVAN, E. K. (2005). «Meaning of aging in women's lives», Journal of Women and Aging, vol. 17, n 3, p. 3-22.

DE BEAUVOIR, S. (1970). La vieillesse: essai, Paris, Gallimard.

DES AULNIERS, L. (1993). «Pillage en douce ou radicalité attentive? L'ethnobiographie en situation de menace», Recherches qualitatives, vol. 9, automne, p. 115-136.

DES AULNIERS, L. (1997). Itinérances de la maladie grave: Le temps des nomades, Paris, L'Harmattan.

DES AULNIERS, L. (2009). La fascination, Nouveau désir d'éternité, Québec, Presses de I'Université du Québec.

FROUCHT-HIRSCH, S. (2005). Le temps d'un cancer: Chroniques d'un médecin malade, Paris, Librairie Vuilbert. 
FUCHS, T. (2001). «Melancholia as a desynchronization: Towards a psychopathology of interpersonal time», Psychopathology, vol. 34, n 4, p. 179-186.

GARNER, D.J. (1999). «Feminism and Feminist Gerontology», Fundamentals of Feminist Gerontology, vol. 11, n²-3, p. 3-12.

GRENIER, A. et J. HANLEY (2007). «Older women and 'frailty', aged, gendered and embodied resistance», Current Sociology, vol. 55, n² 2, p. 211-218.

IONESCO, E. (1963). Le Roi se meurt, Paris, Gallimard.

KUPKE, C. (2005). «Lived time and to live time», Philosophy, Psychiatry, \& Psychology, vol. 12, $\mathrm{n}^{\circ} 3$, p. 199-203.

KUUPPLELOMÄKI, M. et L. SIRKKA (1998). «Cancer patient's reported experiences of suffering», Cancer Nursing, vol. 21, n 5, p. 364-369.

LANTERI-LAURA, G. (1993). «Introduction à I'oeuvre psychopathologique d'Eugène Minkowski», dans MINKOWSKI, E., Études sur la structure des états de dépression: Les dépressions ambivalentes, Paris, Éditions du Nouvel Objet, p. 63-118.

LUSSIER, V. et S. DANEAULT (2006). «Le sujet en souffrance au sein de l'espace médical: au-delà des effractions de la maladie, les effets désubjectivants de la déshumanisation des soins», Revue Québécoise de Psychologie, vol. 26, n 2, p. 39-63.

MAYER, R., F. OUELLET, M.-C. SAINT-JACQUES et D. TURCOTTE (2000). Méthodes de recherche en intervention sociale, Boucherville, G. Morin.

MERRIAM, S.B.(2009). Qualitative Research, A Guide to Design and Implementation, San Francisco, Jossey-Passey.

MINKOWSKI, E. (1933, édition 1995). Le temps vécu: études phénoménologiques et psychopathologiques, Paris, Presses universitaires de France.

M'UZAN, M. D. (1977). De l'art à la mort: itinéraire psychanalytique, Paris, Gallimard.

NELSON, T. D. (2002). «Ageism : Prejudice Against our Feared Future Self», Journal of Social Issues, vol. 2, n 61, p. 207-221.

PAILLÉ, P. et A. MUCCHIELLI (2003). L'analyse qualitative en sciences humaines et sociales, Paris, Armand Colin.

RICFUR, P. (1994). «La souffrance n'est pas la douleur», dans J.-M. VON KAENEL et B. AJCHBAUM-BOFFETY, Souffrances, corps et âme, épreuves partagées, Paris, Éditions Autrement, p. 58-70.

RICCEUR, P. (1950, édition 2009). Philosophie de la volonté, Paris, Éditions Points.

RICCEUR, P. (2000). La mémoire, I'histoire, I'oubli, Paris, Seuil.

SAARADON ECK, A. (2009). «Le cancer comme inscription d'une rupture biographique dans le corps», dans COUSSON-GÉLIE, E., E. LANGLOIS et M. BARRAULT, Faire face au cancer. Image du corps, image de soi, p. 285-311.

STATISTIQUES CANADA (2007). Portrait de la population canadienne en 2006, selon l'âge et le sexe, Recensement de 2006, Ottawa.

THOMAS, C. (2004). Souffrir, Paris, Rivages poche. 
THOMAS, L.-V. et L. DES AULNIERS (1992). «Cette brèche à colmater? Ruptures entre la vie et la mort et tentatives d'intégration», Frontières, vol. 4, no 3, p. 5-11.

WYLLIE, M. (2005). «Lived time and psychopathology», Philosophy, Psychiatry \& Psychology, vol. 12, n 3, p. 173-185.

\section{NOTES}

1 Il est difficile d'interpréter comment les femmes que nous avons rencontrées donnent sens à leur passé en général et comment cela se lie à leur souffrance puisque ce genre d'investigation approfondie demande beaucoup de temps. Or, cela est très ardu à réaliser en deux entrevues qui portent sur plusieurs thèmes. Cette étude ouvre donc certaines pistes de réflexion à ce sujet, mais il serait pertinent d'explorer cette dimension plus en profondeur dans le cadre d'études ultérieures, tel que des études de cas, par exemple.

2 Nous indiquons tout au long de l'analyse, le numéro attribué à la participante dont la citation est présentée suivi de celui de l'entrevue de laquelle cet énoncé est tiré ( 1 pour première entrevue, 2 pour deuxième entrevue) à la suite des verbatims. 